\title{
Microscopic Haematuria in ANCA-Associated Vasculitis with Glomerulonephritis During Treatment and Remission
}

Anoek AE de Joode ${ }^{*}$, Stephanie JM Middelkoop, Jan Stephan F Sanders and Coen A Stegeman

Internal Medicine, Department of Nephrology, University Medical Center Groningen, University of Groningen, Groningen, the Netherlands

*Corresponding author: Anoek A. E de Joode, MD, Internal Medicine, Department of Nephrology, University Medical Center Groningen, University of Groningen, Groningen, the Netherlands, Tel: +31503616161; E-mail: a.a.e.joode@umcg.nl

Received date: June 10, 2016, Accepted date: August 30, 2016, Published date: September 05, 2016

Copyright: ( $2016 \mathrm{E}$ de Joode AA, et al. This is an open-access article distributed under the terms of the Creative Commons Attribution License, which permits unrestricted use, distribution, and reproduction in any medium, provided the original author and source are credited.

\begin{abstract}
Background: ANCA-associated small vessel vasculitides (AAV) are prone to cycles of relapse and remission. Renal involvement manifests as glomerulonephritis with microscopic haematuria, red blood cell casts, proteinuria and variable decrease in renal function. Remission of renal vasculitis is defined as stabilization in serum creatinine (Creat) and resolution of haematuria while controversy exists about persistence of haematuria (during apparent disease-remission) since it may indicate smouldering disease-activity or should be considered as renal flare.
\end{abstract}

Objective: To clarify the course of haematuria after diagnosis and induction therapy and its possible predictive value of long term renal function.

Design: Retrospective cohort study.

Participants: 96 consecutive AAV-patients with renal involvement diagnosed and treated with systemic AAV between $1^{\text {st }}$ of January 2000 to $31^{\text {th }}$ December 2007 were followed for 60 months.

Main measures: Collected data were Creat, CRP $(\mathrm{mg} / \mathrm{ml})$, eGFR $\mathrm{ml} / \mathrm{min} / 1.73 \mathrm{~m}^{2}$, creatinine-excretion in collected $24 \mathrm{~h}$ urine (Ucreat/24 h), proteinuria (Uprot), ratio of proteinuria/ creatinine in $24 \mathrm{~h}$ urine (Uprot/creat) and haematuria. Data were analysed for the complete study population and compared for MPO-ANCA and PR3-ANCA.

Key results: At twelve months after diagnosis, haematuria was no longer detectable in $92 \%$ of all patients. In the PR3-ANCA group, haematuria disappeared after 13 months, while in the MPO-ANCA group haematuria persisted in $19 \%$ of the patients. On average, haematuria disappeared almost simultaneously with stabilisation of the renal function.

Conclusion: Haematuria persists for many months after diagnosis and disappears usually simultaneously with stabilisation of kidney function. There was no relation between persistence of haematuria for over 12 months and renal function during follow up. Haematuria probably acts as a sensitive marker for absence of inflammatory glomerular disease activity in most patients with systemic AAV and renal involvement. It is disappearance coincide with stabilisation of renal function and remission of the disease in almost all patients. However, if it persists, it is not predictive for worsening renal function nor for relapse. Proteinuria does not seem to be a reliable marker for renal disease remission.

Keywords: Haematuria; Prediction; ANCA; Vasculitis; Relapse

\section{Introduction}

The small-vessel vasculitides consist of a heterogeneous group of uncommon systemic multi-organ disorders that are prone to cycles of remission and relapse [1]. These vasculitides include Granulomatosis with Polyangiitis (GPA), Microscopic Polyangiitis (MPA), and eosinophilic Granulomatosis with Poly-Angiitis (eGPA) [1]. These diseases are strongly associated with the presence of antineutrophil cytoplasmic autoantibodies (ANCA): more than $90 \%$ of patients have ANCA against proteinase 3 (PR3-ANCA) and myeloperoxidase (MPO-ANCA) [1-4]. Renal involvement is the most common severe manifestation in patients with ANCA-associated vasculitis (AAV), manifesting as glomerulonephritis with microscopic haematuria, red blood cell casts, proteinuria and a variable level of renal failure [1-3].

Since AAV is a chronic disease with disease-free periods and relapses, therapy usually starts with induction therapy based on cyclophosphamide and corticosteroids, inducing remission in weeks to months in most patients $[5,6]$. Remission of renal vasculitis is defined as decrease or resolution of clinical disease manifestations and subsequent stabilisation in serum creatinine; thoughts about the resolution of haematuria and/or proteinuria are less clear [1]. It is suggested that the persistence of haematuria in AAV patients (in apparent remission) reflects a kind of smouldering disease-activity or should be considered as a renal flare that might benefit from increasing immunosuppressive therapy [1]. 
Page 2 of 4

However, controversy about the meaning or the time course of haematuria in patients with ANCA-associated vasculitis exists. The aim of this study was, therefore, to clarify the course of haematuria after diagnosis and induction therapy and its possible predictive value on long-term renal function. Since PR3- and MPO-ANCA associated vasculitides have distinct clinical and Histopathological differences, comparison was made between these different entities.

\section{Methods}

\section{Study population}

We studied 96 consecutive patients newly diagnosed with systemic AAV and renal involvement and treated in the period $1^{\text {st }}$ of January 2000 to $31^{\text {th }}$ December 2007 within our hospital. Patients were followed on regular outpatient clinic visits for at least 18 months following start of therapy; thereafter follow up was completed 60 months after diagnosis, until death or loss to follow-up.

Due to insufficient follow-up data on urinary parameters in the first 18 months three patients were removed from the analysis leaving 93 patients in the final analysis.

\section{Study design and definitions}

During routine follow up, data were collected at different moments during the disease period, i.e. 0,2 and 4 weeks and from month 2-18 at each visit on the outpatient clinic (at least every three months). Collected data were serum creatinine, CRP $\mathrm{mg} / \mathrm{l}$ and 24 hour urine collection for creatinine and protein (Ucreat mmol/24 h, Uprot gr/24 h). The Uprot/creat was calculated with the formula: proteinuria/ creatinine-excretion, gr/24 h, in collected $24 \mathrm{~h}$ urine. In addition, a freshly voided urine specimen was checked for presence of haem, and if positive, microscopic analysis of the urinary sediment was performed to quantify haematuria. A number less than 5 erythrocytes per high powered field (corresponds to approximately 25/ul) was regarded as non-significant or negative while 5 or more per field was regarded as significant haematuria. Malignancy of urogenital tract was ruled out in all cases. Remission of haematuria was defined as a normal value; it means a negative stick or less than 5 erytrocytes/htf, for at least 3 consecutive months or a longer lasting course with one single abnormal value. The first of these 3 normal values was defined as the 'month of remission'. Serum creatinine was used to define a clinical stable renal function. Renal function was considered stable at the lowest serum creatinine value and a stable serum creatinine for 3 consecutive months combined with stabilised clinical characteristics. Proteinuria was assessed as the ratio of urinary protein $(\mathrm{g} / \mathrm{l})$ and creatinine $(\mathrm{mmol} / \mathrm{l})$. Remission of Uprot $/$ creat $(\mathrm{g} / \mathrm{mmol})$ was defined as the lowest value during follow up followed by at least 3 months of stable values. Long term persistence of haematuria, proteinuria and unstable serum creatinine were defined as not reaching the definition for remission at 12 months. The course of the haematuria within the whole study population, as well as the differences in haematuria course for MPO-ANCA and PR3-ANCA patients were analysed. The same was done for the course of serum creatinine and the ratio of Uprot/ Creat. Third, the moments of remission between serum creatinine and haematuria as well as serum creatinine and the ratio Uprot/Creat were compared. Finally, a comparison was made for the long term renal function. Primary outcome of this study was the course of haematuria during treatment; secondary outcomes were renal function (serum creatinine), proteinuria and change in renal function during follow up.

\section{Statistical analyses}

Descriptive statistics were generated and analysed using SPSS20. Outcomes were analysed with Mann-Whitney U test or Chi-square test. The course of haematuria, Creat and the Uprot/Creat were analysed with Kaplan Meier curves and the Log Rank test was used for comparison of groups.

\section{Results}

\section{Baseline characteristics}

Fifty-six patients were PR3-ANCA positive (58\%), 37 were MPOANCA-positive (39\%) one was HNE-positive and two patients were ANCA negative. Baseline characteristics were compared for PR3ANCA and MPO-ANCA positive patients (Table 1). Of all the patients, 65 were male (68\%). Median age was 63 (range 51.3-72.5) years. In 38 patients $(40 \%)$ the diagnosis was renal biopsy-proven. Twenty-four patients (26\%) died within the 60 months of follow-up after diagnosis of which $15(16 \%)$ during the first 18 months after diagnosis. Significant differences between PR3-ANCA positive and MPO-ANCA positive patients as expected, were found regarding sex, age, serum creatinine, eGFR, clearance, CRP, Ucreat/24 h and Uprot/Creat.

\begin{tabular}{|l|l|l|l|}
\hline & MPO-ANCA & PR3-ANCA & \multirow{2}{*}{ p-value } \\
\cline { 2 - 4 } & $\mathbf{( n = 3 7 )}$ & $\mathbf{( n = 5 6 )}$ & \\
\hline Sex $(\mathrm{M} / \mathrm{F})$ & $19 / 18$ & $44 / 12$ & $0.006 \mathrm{~b}$ \\
\hline Age (years) & $66(56-75)$ & $60(45-70)$ & $0.03 \mathrm{~b}$ \\
\hline Haematuria (\%) & 100 & 100 & 0.72 \\
\hline Creat $(\mu \mathrm{mol} / \mathrm{l})$ & $257(144-626)$ & $119(100-328)$ & $0.007 \mathrm{~b}$ \\
\hline eGFR (ml/min/1,73 $\left.\mathrm{m}^{2}\right)$ & $17(7-34)$ & $56(17-69)$ & $0.001 \mathrm{~b}$ \\
\hline Clearance & $20(7-43)$ & $59(13-85)$ & $0.002 \mathrm{~b}$ \\
\hline CRP (mg/l) & $48(14-120)$ & $120(50-169)$ & $0.001 \mathrm{~b}$ \\
\hline UCreat (mmol/24 u) & $7.4(5.2-10.2)$ & $9.9(5.7-12.8)$ & $0.05 \mathrm{~b}$ \\
\hline Proteinuria (g/24 u) & $1.00(0.3-1.8)$ & $0.8(0.5-1.4)$ & 0.5 \\
\hline $\begin{array}{l}\text { Ratio Uprot/Creat } \\
\text { mmol) }\end{array}$ & $\begin{array}{l}0.19 \\
(0.05-0.42)\end{array}$ & $\begin{array}{l}0.09 \\
-0.16)\end{array}$ & $0.027 \mathrm{~b}$ \\
\hline
\end{tabular}

Table 1: Baseline patient characteristics. A-median (IQR) unless otherwise specified; B-Statistically significant

\section{Haematuria}

At baseline haematuria was present in all patients. At 1, 3, 6, 12 and 18 months of follow up, haematuria was present in 58\%, 28\%, $22 \%$, $10 \%$ and $6 \%$ of the patients respectively. Haematuria had disappeared in $72 \%$ and in $92 \%$ after 6 and 12 months respectively. When comparing PR3-ANCA and MPO-ANCA at 6 and 12 months, haematuria had disappeared in $80 \%$ and $98 \%$ for PR3-ANCA and $57 \%$ and $81 \%$ in MPO-ANCA (log rank $\mathrm{p}=0.005)$. After 13 months, in all PR3-ANCA positive patients haematuria was no longer detectable, while it persisted in $19 \%$ of the MPO-ANCA positive patients (Figure $1)$. 
Citation: de Joode AAE, Middelkoop SJM, Sanders JSF, Sanders CA (2016) Microscopic Haematuria in ANCA-Associated Vasculitis with

Page 3 of 4
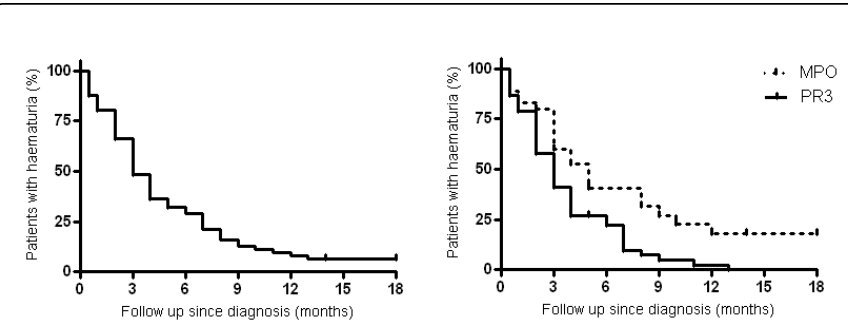

Figure 1: A: Survival curve of haematuria for complete study population, 1B :PR3- versus MPO-ANCA positive patients ((Log rank $\mathrm{p}=0.003)$.
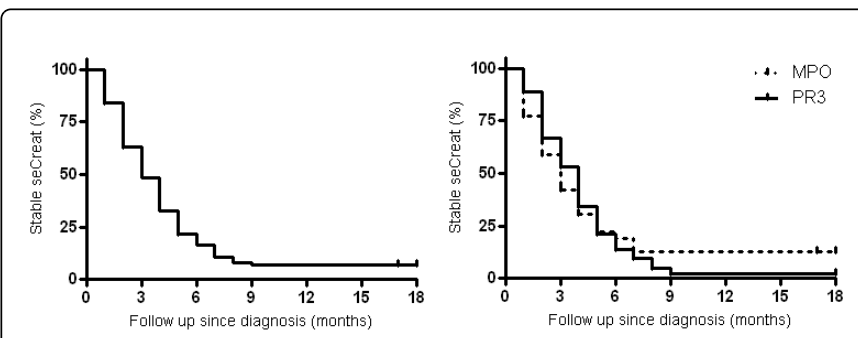

Figure 2: A) Survival cure of, serum creatinine for complete study population; 2B: PR3- versus MPO-ANCA positive patients.
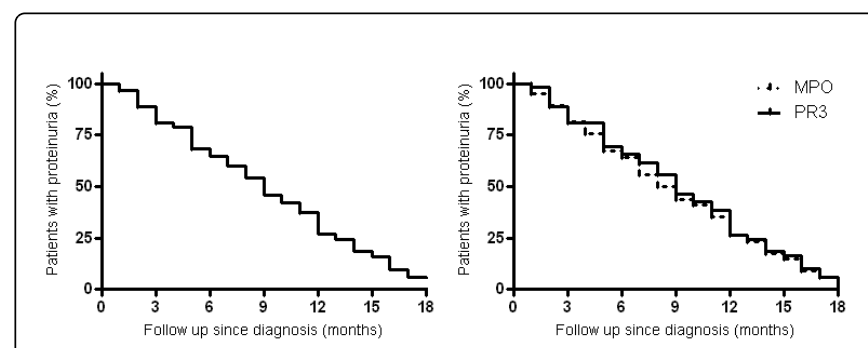

Figure 3: A) Survival curve of Uprot/Creat in collected 24h urine for complete study population; 3B. PR3- versus MPO-ANCA positive patients.

\section{Serum creatinine}

Within 6 and 12 months after diagnosis, serum creatinine stabilized in $84 \%$ and $94 \%$ respectively. Comparing PR3-ANCA and MPOANCA at 6 and 12 months, serum creatinine was stable in $86 \%$ and $96 \%$ for PR3-ANCA and $80 \%$ and $88 \%$ in MPO-ANCA respectively $\log$ rank $\mathrm{p}=0.90$ ( Figure 2).

\section{Haematuria and serum creatinine}

The mean difference between a stable kidney function and the disappearance of haematuria was 0.036 months (SD 2.79). For the PR3-ANCA group this was 0.42 months (SD 2.72) and for MPOANCA -0.98 months (SD 2.80). This indicates that, after diagnosis, on average the haematuria disappearance and stabilisation of serum creatinine coincide in time. A crosstab was generated to compare the patients with haematuria and unstable serum creatinine for more than 12 months. No correlation between these two parameters was found (Fisher's Exact $\mathrm{p}=1.000$ ); therefore no further prediction can be made based on the findings of the first 12-18 months after diagnosis. Haematuria nor the persistence after 6 and 12 months were predictors for renal function over time nor for relapse until 60 months after diagnosis, not even when separately analysed for ANCA-specificity.

\section{Proteinuria}

After a follow-up period of 6 and 12 months from diagnosis, the Uprot/creat reached stabilisation in $35 \%$ and $74 \%$ of the patients respectively. Comparing PR3-ANCA and MPO-ANCA at 6 and 12 months, Uprot/creat was stable in $34 \%$ and $72 \%$ for PR3-ANCA and $36 \%$ and $79 \%$ in MPO-ANCA respectively log rank $\mathrm{p}=0.88$ (Figure 3 ).

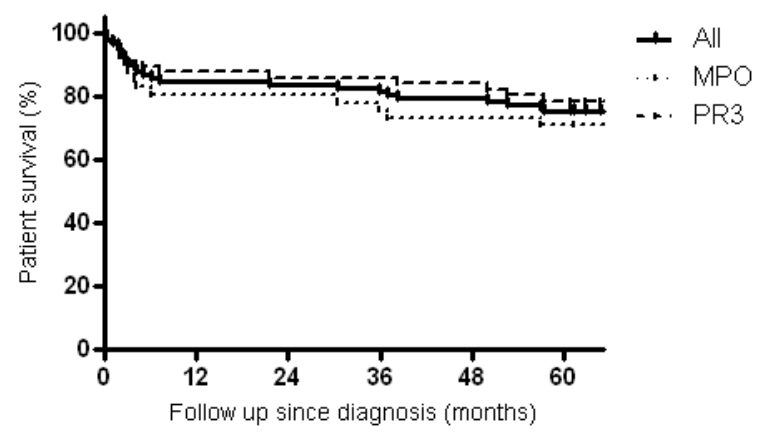

Figure 4: Survival curve for complete study population.

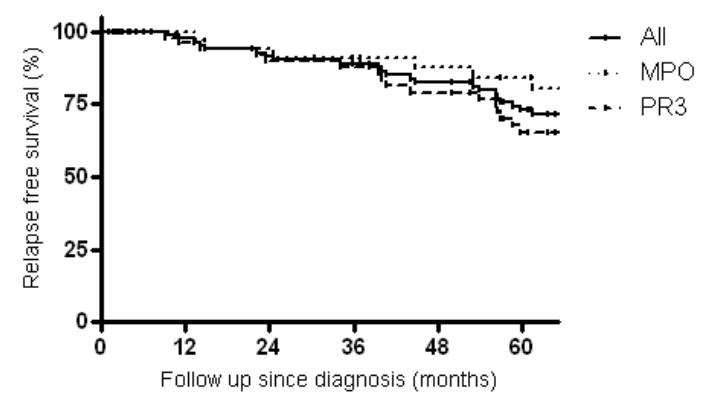

Figure 5: Relapse free survival curve for complete study population

\section{Proteinuria and serum creatinine}

The mean difference between a stable kidney function and the stable Uprot/creat ratio was -6.1 months (SD 4.93) for the total study population. This indicates that serum creatinine stabilises on average about 6 months earlier than proteinuria does. There was no difference between the PR3-ANCA group (-6.0 months (SD 4.9)) and the MPOANCA group (-6.4 months, SD 5.0). Patients survival, relapse-free survival and long-term renal function. During long term follow up, survival rates were $88 \%$ at 12 months and $75 \%$ at 60 months. There was no difference in patient survival between PR3 and MPO-ANCA positive patients, $\mathrm{p}=0.36$ (Figure 4 ).

At 60 months, a third of all patients had experienced a relapse: as expected, these relapses happened more often in the PR3-ANCA positive patients, $16 \%$ in MPO-patients $(\mathrm{n}=6)$ and $30 \%$ in PR3-patients $(\mathrm{n}=17)$, with a trend towards significance, $\mathrm{p}=0.12$ (Figure 5). During 
long term follow up, overall renal function did not change significantly over time without difference for ANCA-specificity or experiencing relapses (Figure 6).

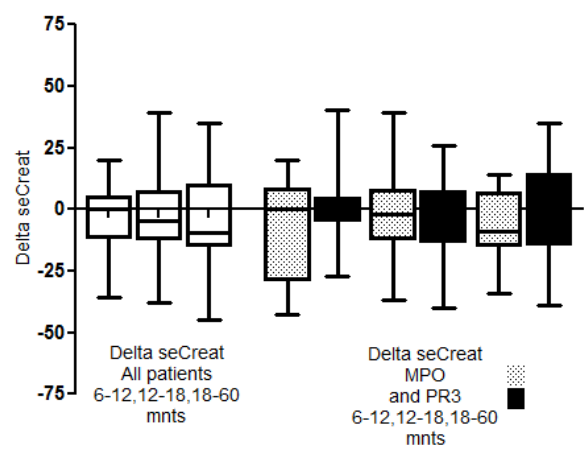

Figure 6: Delta serum creatinine 6-12, 12-18 and 18-60 months.

\section{Discussion}

In this retrospective study of 96 patients with pauci-imune glomerulonephritis it was questioned whether microscopic haematuria in patients with renal vasculitis in clinical remission could represent chronic glomerular injury from prior episodes of disease-activity or may represent new glomerular pathology [1]. We found that in general, haematuria disappears between three and six months after diagnosis and start of induction therapy and that the disappearance coincides in time with stabilisation of renal function. Nevertheless, as reflected by persisting proteinuria, microscopic injury may persists for a longer period. During follow up, persistence of haematuria is neither associated with worsening renal function nor predictive for relapse.

In prior reports, Magrey et al. suggested that a subset of patients with GPA and glomerulonephritis may have persistent microscopic haematuria from glomerular injury and not from active disease, stating that patients with GPA and glomerulonephritis probably achieved enduring remission, allowing withdrawal of medication, despite continued microscopic haematuria with or without RBC casts [3]. Franssen et al. described resolution of haematuria within four months after start of treatment in a large cohort study of MPO-ANCA positive patients. None of the treated patients who developed chronic renal failure had haematuria at any time during follow-up but in addition each of the relapses encountered during follow up was associated with recurrence of haematuria [7]. In our cohort of 96 patients with systemic AAV and renal involvement, after follow-up of 6 months, haematuria had disappeared in $72 \%$ of the whole study population. However, only in the PR3-ANCA positive patients, haematuria became negative at follow-up of 13 months, while in the MPO-ANCA positive patients, haematuria persistent in a small group (19\%) of patients. There was no association with the persistence of haematuria and decline in renal function or relapse in these patients.

However, this difference between PR3-ANCA and MPO-ANCApositive patients was significant, $\mathrm{p}=0.003$, which again may underscore the conclusion that PR3-AAV and MPO-AAV are distinct diseases. On average the haematuria disappeared almost simultaneously with stabilisation of the renal function and only a small minority had ongoing haematuria 6 months after diagnosis. The proteinuria persisted for about 6 months longer compared to stabilisation of the renal function. On these grounds, one can conclude that the disappearance of haematuria can act as a marker for stabilisation of renal function and absence of on-going inflammatory disease activity. Proteinuria therefore cannot be used as a marker for quiescence of renal disease. During long term follow up, haematuria or the persistence appeared not to be of predictive value for renal function nor for relapses and can certainly not act as a guidance for therapy. There are some considerations: a number of missing values during follow up could have influenced the analyses, for instance in defining date of remission in these particular patients. In addition, it is debatable whether the study population is representative for the population of patients with systemic AAV since in our tertiary referral centre mostly complex patients are treated who sometimes did not immediately responded to standard induction therapy. This can have had influence on outcomes. Nevertheless, considering the fact that even complex patients are also included in these analyses, we think that our findings on haematuria, remission and predictive value could be generalizable.

In conclusion, we found that haematuria probably acts as a sensitive marker for absence of inflammatory glomerular disease activity in most patients with systemic $\mathrm{AAV}$ and renal involvement. It's disappearance coincide with stabilisation of renal function and remission of the disease in almost all patients. However, if it persists, it is not predictive for worsening renal function nor for relapse. This means that one should monitor these patients closely, but there is not enough evidence that supports escalation of immunosuppressive medication based on persistence of haematuria alone. Furthermore, since microscopic injury as indicated by proteinuria persists for longer duration, proteinuria does not seem to be a reliable marker for renal disease remission.

\section{References}

1. Geetha D, Seo P, Ellis C, Kuperman M, Levine SM (2012) Persistent or new onset microscopic hematuria in patients with small vessel vasculitis in remission: findings on renal biopsy. J Rheumatol 39: 1413-1417.

2. Neumann I, Kain R, Regele H, Soleiman A, Kandutsch S, et al. (2005) Histological and clinical predictors of early and late renal outcome in ANCA-associated vasculitis. Nephrol Dial Transplant 20: 96-104.

3. Magrey MN, Villa-Forte A, Koening CL, Myles JL, Hoffman GS (2009) Persistent haematuria after induction of remission in Wegener granulomatosis: a therapeutic dilemma. Medicine (Baltimore) 88:315-321.

4. de Joode AA, Sanders JS, Stegeman CA (2013) Renal survival in proteinase 3 and myeloperoxidase ANCA-associated systemic vasculitis. Clin J Am Soc Nephrol 8: 1709-1717.

5. Cattran DC (2012) KDIGO Clinical Practice Guideline for Glomerulonephritis. J Int Soc Nephrol 2:2

6. Slot MC, Tervaert JW, Franssen CF, Stegeman CA (2003) Renal survival and prognostic factors in patients with PR3-ANCA associated vasculitis with renal involvement. Kidney Int 63: 670-677.

7. Franssen CF, Stegeman CA, Oost-Kort WW, Kallenberg CG, Limburg PC, et al. (1998) Determinants of renal outcome in anti-myeloperoxidaseassociated necrotizing crescentic glomerulonephritis. J Am Soc Nephrol 9:1915-1923. 